\title{
A Mobility Performance Assessment on Plug-in EV Battery
}

\author{
Seyed Mohammad Rezvanizanian, Yixiang Huang, Jiang Chuan, and Jay Lee \\ Center for Intelligent Maintenance System, University of Cincinnati, Cincinnati, Ohio, 45220, USA \\ rezvansd@mail.uc.edu \\ huangyx@mail.uc.edu \\ jiangcn@mail.uc.edu \\ lj2@mail.uc.edu
}

\begin{abstract}
This paper deals with mobility prediction of $\mathrm{LiFeMnPO}_{4}$ batteries for an emission-free Electric Vehicle. The data-driven model has been developed based on empirical data from two different road types - highway and local streets -and two different driving modes - aggressive and moderate. Battery State of Charge $(\mathrm{SoC})$ can be predicted on any new roads based on the trained model by selecting the drving mode. In this paper, the performance of Adaptive Recurrent Neural Network (ARNN) and regression is evaluated using two benchmark data sets. The ARNN model at first estimates the speed profile of the new road based on slope and then both slope and speed is going to be used as the input to estimate battery current and SoC. Through comparison it is found that if ARNN system is appropriately trained, it performs with better accuracy than Regression in both two road types and driving modes. The results show that prediction SoC model follows the Columbcounting SoC according to the road slope. ${ }^{1}$
\end{abstract}

\section{INTRODUCTION}

Concerns with fuel cost, oil shortages, air pollution, and higher fuel economy standards have driven the rapid rise of more fuel-efficient vehicles and Electric Vehicles (EV). The future of transportation is being propelled by a fundamental move to green and more efficient electric drive systems. However, Electric vehicles still represent a small part of the worldwide market. For example, EVs account for just over $1 \%$ of the passenger car market at present (Shafiei \& Williamson, 2010). With recent major advancements in battery technology, however, more electric vehicles are anticipated to enter the market within the next few years.

S. Mohammad Rezvanizaniani et al. This is an open-access article distributed under the terms of the Creative Commons Attribution 3.0 United States License, which permits unrestricted use, distribution, and reproduction in any medium, provided the original author and source are credited.
New concepts and technologies need to be developed to launch electrically chargeable vehicles suited for both individual and public mobility and for goods distribution in urban areas. Electrically charged vehicles provide many benefits in metropolitan areas, such as very low (plug-in hybrid electric vehicle - PHEV) to zero (battery electric vehicle - BEV) tailpipe emissions and reduced noise.

Although battery technology has developed considerably in recent decades, the main drawback of electric vehicles still remains: the low range of such vehicles in comparison to their gas-powered counterparts. Engineers and EV designers are challenged to improve EV mobility performance with existing battery power. The lead-Acid battery - and its variants- is the dominant battery in the automotive industry due to its low manufacturing price, high $\mathrm{C}$-rate discharge and good low temperature performance. However, the low energy density of these batteries makes them unsuitable for hybrid and electric vehicle applications. Currently, car manufacturers are motivated to utilize Ni-MH and $\mathrm{Li}$-ion batteries because their high energy density and cycle life that satisfy the requirements of an electric vehicle. Therefore accurate estimation of the $\mathrm{SoC}$ of a high capacity energy storage system can improve energy management and efficient utilization of electric vehicle by optimizing performance, lengthening the cycle life and providing more useful information for the driver.

The output power from the battery depends on a number of factors, such as discharge current rate, internal resistance, battery age, environment temperature and historical usage (Meissner \& Richter, 2003; Ulrich, 2012). All above factors influence $\mathrm{SoC}$ in linear and non-linear ways. Therefore, a number of diverse techniques have been proposed to calculate or estimate the SoC of a battery each of which as its relative advantages and constraints, as reviewed by (Piller, Perrin, \& Jossen, 2001; Rodrigues, Munichandraiah, \& Shukla, 2000; Zhang \& Lee, 2011). However, all of these techniques attempt to monitor and measure the $\mathrm{SoC}$ at the current time. Applying prognostics algorithms can help 
engineers predict battery conditions as well. Fully informed travelers and increased productivity of energy storage devices are the requirements for enabling the intelligent mobility of an electric vehicle. Due to the broad availability of low cost wireless communication systems, related information flows constantly and flawlessly from each source to all interested users. Using these systems provides an opportunity for drivers to obtain directions and optimize their route before starting a trip. Moreover, these devices can provide the raw data necessary for prognostics algorithms to predict the conditions of the battery as well.

This paper investigates new methods of using machine learning techniques and prognostics algorithms to predict the battery SoC of an EV prior to starting a trip, which is based on the route selected and historical driving behavior by the driver. Section 2 gives an overview of why the market is looking for SoC estimation and EV battery performance prediction. The details of empirical testing on EV are presented in section 3. Section 4 provides the methodology and details of algorithms used for prediction. The details of the data analysis, including feature extraction and SoC estimation, are presented in section 5. Finally, the results and conclusion are discussed in section 6 and 7.

\section{PROBLEM STATEMENT}

One of the most important barriers in acceptance of electric mobility is a range of electric vehicle. It has been evaluate the effects of low range resources of electric vehicles, as a significant feature for users' purchase intentions by market experts and prospective customers. As it has been mentioned before new communication technology provides much more data to the interested user. A GPS can provide some basic route options including local streets, highways or combination of both with an estimation of travel time. However, such estimations are not tailored for the specific needs of EV users. There is no prediction of energy consumption for suggested routes, or any inclusion of

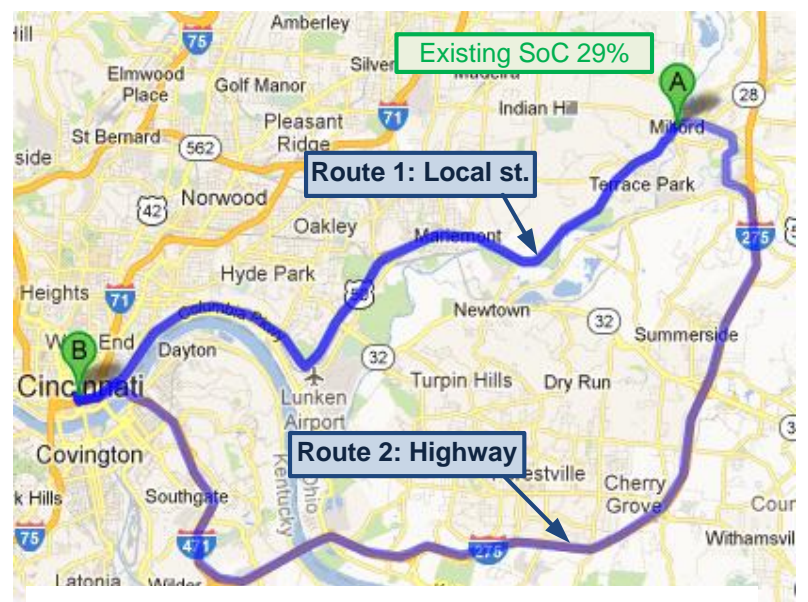

Figure 1. Two different types of road (highway and local streets) driving behavior or road conditions. Route suggestions based on this information would go a long way towards alleviating potential range anxiety that is one of the major barriers for EV adoption.

In battery prognostics, since it is a relatively new field of interest, there are few works that can fit inside the requirements. For example, (Gonder, Markel, Thornton, \& Simpson, 2007) found that around 95\% of daily driving can be achieved with 100 miles of electric range by studying a set of vehicles for thirty weekdays each. Correspondingly, studies by (MacLean \& Lave, 2003) and (Sioshansi \& Denholm, 2009) on hybrid vehicles have determined that about three-quarters of travel miles could be powered by electricity. The main issue with such studies is that customers desire a vehicle that fulfills their own diverse needs over time, not the needs that are dictated by statistics or the average profile. In general, changing the charge status of the battery is a complex process, resulting from the interaction of several factors. Among these factors, discharging current rate is the most significant, and it depends on several car resistances. For mechanical power generation of a vehicle, the electric motor should be able to provide enough power to accelerate and propel the EV if it encounters any resistance. There are three significant forms of resistance for a vehicle: tire friction, aerodynamic resistance and gravity resistance (Fodor, Enisz, Doman, \& Toth, 2011; Khaled, Harambat, Yammine, \& Peerhossaini, 2010; Shukla, Aricò, \& Antonucci, 2001). These forms of resistance are directly influenced by vehicle speed and road slope. Moreover the driver's driving habits affected battery performance. Even the same maximum speed on a road with different accelerations can have different effects on the charge of battery. In this paper this term is called "driving mode" and is defined it as acceleration and braking. Hence this paper looks for a method to predict SOC based on three factors:

\section{Vehicle Speed \\ 2. Road Slope (Terrain type) \\ 3. Driving Mode}

Researchers have established different techniques for formulating a relationship between SoC and these factors. In (He, Xiong, Zhang, Sun, \& Fan, 2011) an improved Thevenin model and utilized Extended Kalman Filter were used to calculate SoC for an EV which was driving at variable speeds. In (He, Xiong, \& Guo, 2012; Xu, Wang, \& Chen, 2012) the battery was modeled with combination of Extended Kalman Filters and Fuzzy Neural Network to give an approximation of SoC. Some researchers attempted to determine realistic driving conditions and their effects on battery performance. In (Adornato, Patil, Filipi, Baraket, \& Gordon, 2009; Lee, Baraket, Gordon, \& Filipi, 2011) the authors classified practical driving to identify a charging model for each category of driving mode. In (Marina de Queiroz Tavares, 2010) GPS tracker is using to monitor 
where the EV is charging and where it goes in order to recognize driving behavior. What's missing is how to define the relationship between these factors and mobility.

In fact, the inclusion of all these variables would complicate the findings of SoC prediction. For clarification purposes, this paper will focus on one variable and its effect on battery performance, energy efficiency, and long-term operation on battery Remaining Useful Life (RUL). Hence, several tests have been designed on the EV to be tested on different roads with different slopes and see how the slope can affect battery performance.

\section{EXPERIMENTAL DESCRIPTION}

The best approach to represent real-world driving patterns is to test an EV on specific routes and collect voltage and current from battery and speed from car. A major drawback of this approach is that it is difficult to justify the value that can be derived from such costly experiments. Because of the sporadic nature of driving cycles and the wide distribution of driving conditions, obtaining meaningful information from field testing for accurate analysis is very difficult. Researchers previously summarized statistical results from field tests to offer some help in understanding battery performance (Huang, Tan, \& He, 2011; Lee et al., 2011; Liaw \& Dubarry, 2007; Montazeri-Gh, Fotouhi, \& Naderpour, 2011). According to the literature, road type, traffic road slope, traffic and driving mode have major affects on battery state of charge and energy consumption during a trip; however, there are some other factors such as ambient temperature, humidity, and charging intervals that can affect on battery life on long term operation. Since considering all factors make the problem very complex and difficult to solve, in this paper we set these factors to constant values and conduct the test on just different road types and with driving modes to investigate battery performance in diverse conditions. Data has been collected from a Chevy Equinox (Figure 2), which has been converted from a regular gas powered car to an emissions-free Electric Vehicle by AMP. This car has been equipped with $\mathrm{LiFeMnPO}_{4}$ battery from the GBSystem. The basic specifications of the EV are summarized in Table 1.

\begin{tabular}{l|l|l}
\hline Characteristics & Range & Unit \\
\hline Car Range & $90-100$ & Miles \\
\hline Max Speed & 90 & $\mathrm{mph}$ \\
\hline Number of Cells & 108 & \\
\hline Cell connection & in-series & \\
\hline Total Battery Energy & 40 & $\mathrm{kWh}$ \\
\hline Battery type & LiFeMnPO $_{4}$ & \\
\hline Nominal Capacity & 100 & A.h \\
\hline
\end{tabular}

Table 1. Chevy Equinox and battery pack specification
To make the condition of the tests as stable as possible all tests have been done using a single car. In each test two persons were in the car (the driver and one passenger were to control data collection). The condition of test is based on two road types: highways and local streets. Both road types were selected to have some uphill and downhill conditions to observe battery performance in both conditions.

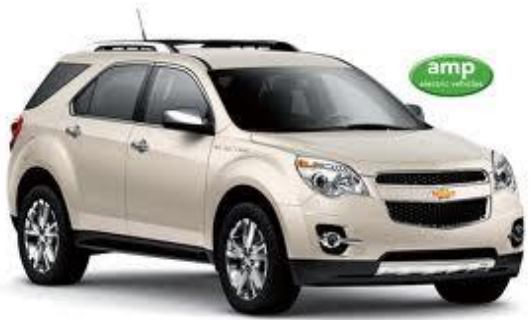

Figure 2. Chevy Equinox electric vehicle

\section{METHODOLOGY}

One of the most proficient ways of solving a multifaceted problem is to decompose it into simpler elements, in order to make it more understandable and more manageable (Bo, Zhifeng, \& Binggang, 2008). In addition, simple elements may be assembled to produce a complex system. One approach for achieving this is using Networks. All networks consist of nodes and connections, where the nodes can be considered to be computational units. Nodes receive inputs and apply some mathematical processes to attain an output. This processing might be very straightforward (such as summing the inputs), or quite complex.

The Neural Network (NN) techniques are one of the common methods, which typically consist of inputs that are multiplied by weights (strength of the particular signals), and then computed by a mathematical function that represents the activation of the neuron. Another function computes the output of the artificial neuron (sometimes in dependence of a certain threshold). NNs combine artificial neurons in order to find relations among inputs and outputs (Charkhgard \& Farrokhi, 2010). In this paper Adaptive Recurrent Neural Network is implemented on EV data. Recurrent or recursive networks are well suited to time variant modeling applications, such as prediction. This is because temporal knowledge is saved by the network in the form of time-delayed inputs; outputs from one iteration of the model are fed back as inputs into one or more succeeding iterations (Wang, Golnaraghi, \& Ismail, 2004).

In this paper the recurrent network has a feedback link from outputs to the inputs, which serves as the third input layer. This layer is illustrated in Figure 3. However, in some cases this recurrent network can be applied from the hidden layer to the context (Wang et al., 2004). This network has three input nodes (slope, speed and previous step of current), three layers and one output. The nodes in the input simply send out input values to the hidden layer. For the nodes in the hidden layers, hyperbolic tangent sigmoid functions 
have been selected as their transfer functions, while a linear function is assigned to the output node. By training the model, the connection weights can be adjusted in the algorithm. The higher the weight of an artificial neuron the stronger the input is; meaning that this specific input is more significant (Abolhassani Monfared et al., 2006; Julka et al., 2011). Eq. (1) shows the function of output I at time $\mathrm{k}$ of three inputs where $\mathbf{p}$ is the slope of the road; $\mathbf{s}$ is the speed of the car; and $\mathbf{I}$ is the current at one step ago.

$$
I(k)=f(P(k-1), S(k-1), I(k-1))
$$

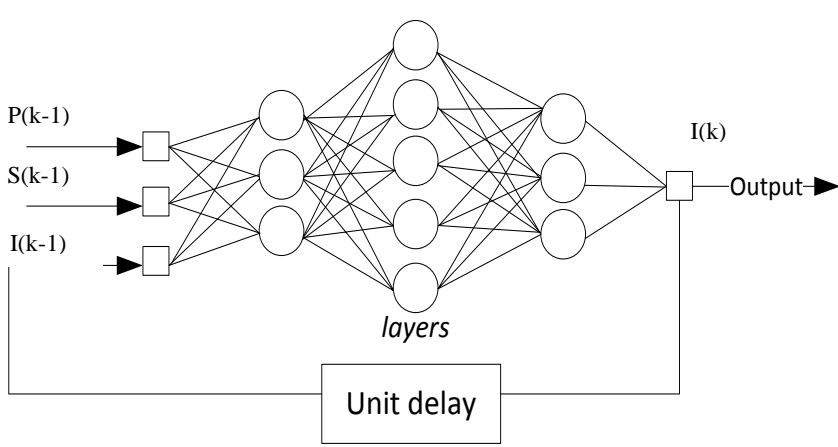

Figure 3. Adaptive Recurrent Neural Network Concepts

A Linear Regression method has been applied on the data to benchmark the accuracy of results with ARNN. In the regression process, a linear model has been built between the inputs and outputs by applying training data. The model is then tested by new inputs and tested with the measured output to calculate error.

\section{DATA ANALYSIS AND DISCUSSION}

\subsection{Feature extraction}

In addition to the aforementioned methods, it is necessary to find the operational parameters that change with different driving modes in order. The accuracy of the battery performance estimation and SoC prediction will heavily rely on these so-called features. From the raw data, many features could be extracted, but not all the features will be directly related to the driving behavior. In this paper, driving mode has been classified into two categories: aggressive and moderate. Currently, two main features are extracted from battery current. To have better understanding of the two different driving modes, Figure 4 shows battery current for aggressive and moderate driving modes on the same local streets with the same driver and the same car.

Figure 5 a) and 5 b) illustrate the distribution of current for these two different driving modes on one type of road (local streets). The aggressive driving mode is distributed in a lager range of current: the minimum value is around $-120 \mathrm{~A}$ and the maximum around $200 \mathrm{~A}$. The moderate driving mode has changed, however, to between -85 A to $140 \mathrm{~A}$.

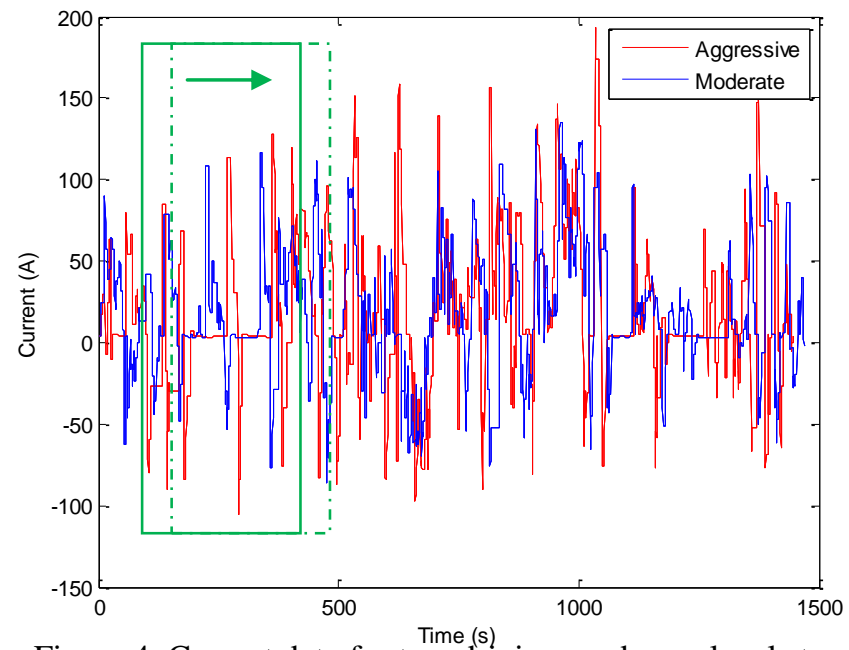

Figure 4. Current data for two driving modes on local streets

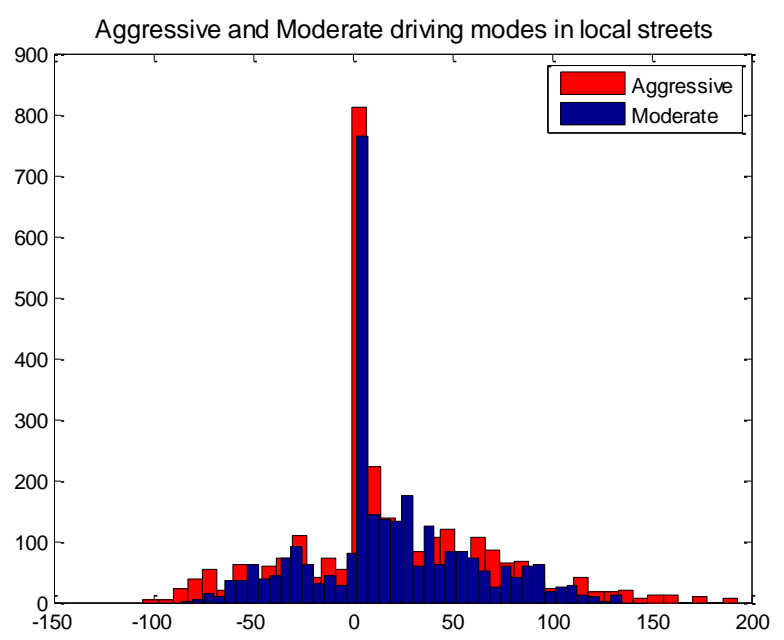

Figure 5 a). Histogram for local streets

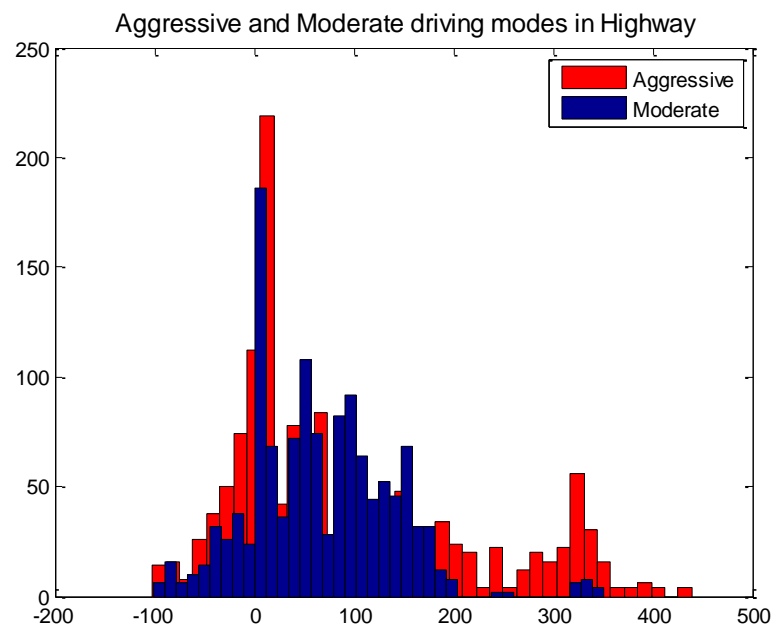

Figure 5 b). Histogram for highway

For highway mode this range has been extended due o the higher speeds reached, since the aggressive driver reaches more than $400 \mathrm{~A}$. The other significant difference is related 
to the high frequency of near zero value for current data. In fact, any time the driver increases speed from zero and implements hard braking, a value close to zero is recorded. This is inevitable when the car is approaching traffic lights, which is a pronounced difference between local streets and highways. Highways do not often contain stop points, that can help us to recognize the route type based on battery current data analysis.

To obtain a mathematical threshold that can help us distinguish between the two driving modes, the standard deviation of both currents has been calculated in the specific window size, by moving the window forward in each by one step in value of standard deviation recorded (Figure 4). Figure 6 represents the results of how the standard deviation for current changes over time. The average of aggressive driving mode standard deviation is 44.3 and the moderate one is 35.6 .

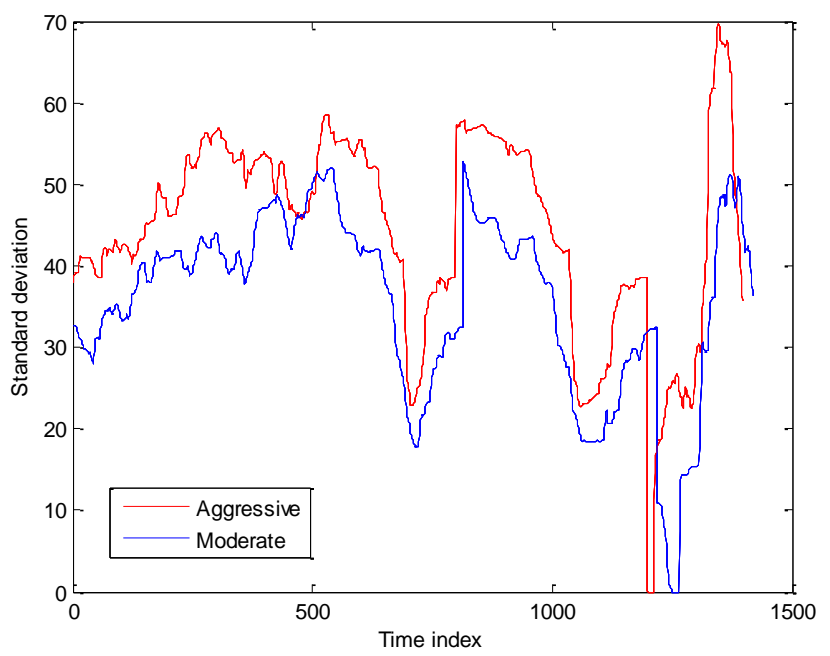

Figure 6. Battery current standard deviation over time

\subsection{SOC Estimation}

The estimation of the SoC of a battery may be a problem of more or less complexity depending on the battery type and on the application in which the battery is used. The most reliable technique to define the SoC for Electric Vehicles during the operation is ampere hour counting (Columb counting) (Zhu, Coleman, \& Hurley, 2004). The idea of balancing the current is reasonable because the charging and discharging cycles are directly related to the supplied or demanded current. If the initial $\mathrm{SoC}$ value is given $\left(\mathrm{SoC}_{0}\right)$, the value of current integral is a straight indicator for the SoC. Eqs. 2 and 3 show this relation for both charge and discharge respectively:

$$
\begin{array}{lr}
S o C=S o C_{0}+\frac{1}{C_{n}} \cdot \int_{t_{0}}^{t}|I| \cdot d t & \text { charge } \\
S o C=S o C_{0}-\frac{1}{C_{n}} \cdot \int_{t_{0}}^{t}|I| \cdot d t & \text { discharge }
\end{array}
$$

Where $C_{n}$ is the nominal capacity of the EV battery pack, $I$ is the battery current and $d t$ is the time interval. It is obvious that SoC is a function of current. Therefore if we can estimate the current of the vehicle before it completes its route then it is possible to calculate SoC. For this purpose we need to figure out how that current is changing based on road condition.

Based on these assumptions, the amount of current demanded from a battery dependent on how fast the car moves, or accelerates and the slope of the road. The main challenge is finding what the relation is between road slope and car speed. Theoretically, the car speed can be independent from the slope. However, in real-world data, we can see that by driving up a hill the speed can be diminished. Actually, there is no definite answer for this question because controlling the speed depends on the driver's decision to keep the speed constant, let it reduce a little or even accelerate. In this paper the car has been assumed that speed is independent from the road slope.

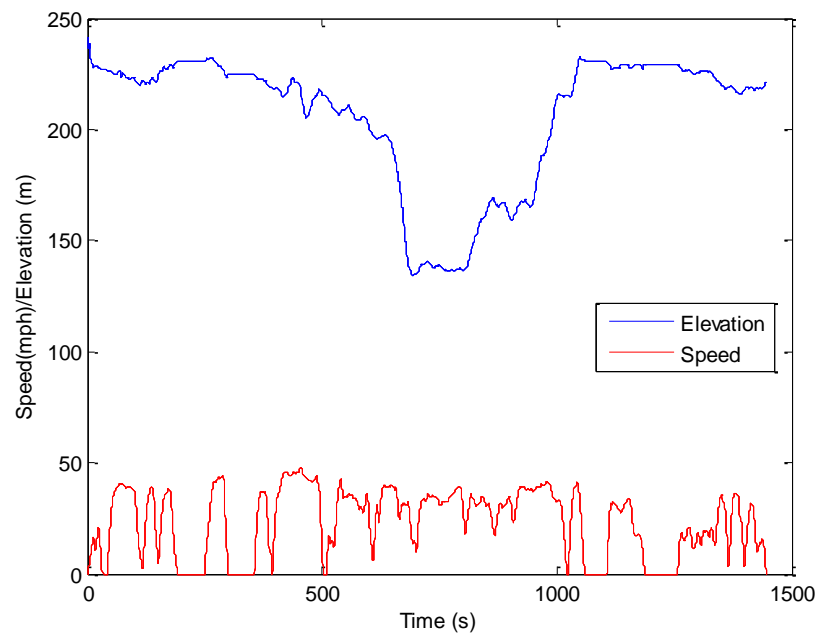

Figure 7. Elevation and Speed in a local street; there are input variables in the model

The ARNN predictor has been constructed based on a feedforward multi-layer neural network with adaptive and feedback links from input nodes. The feedback units reproduce the activations of the nodes from the previous time step, and allow the network to memorize the evidence from the past, which forms a reasoning base for current processing. For the nodes in the hidden layers, the sigmoid activation function has been used and the linear activation function has been selected for the nodes in the output layer. The strategy is to train the model based on the ARNN with speed and slope at the existing time step and current as the output of the model from the previous step as the input variables for two different driving modes (Figure 7) with current as the target (Figure 4 red color). Figure 7 represents the sample input for training, which is road elevation and speed for a local street under an aggressive driving mode 
together. Table 2 summarizes the process of constructing the training data set.

\begin{tabular}{c|c|c|c}
\hline & Road type & Driving mode & $\begin{array}{c}\text { Length of the } \\
\text { road }\end{array}$ \\
\hline 1 & Highway 1 & Aggressive & 10 miles \\
\hline 2 & Highway 1 & Moderate & 10 miles \\
\hline 3 & Local St 1 & Aggressive & 10 miles \\
\hline 4 & Local St 1 & Moderate & 10 miles \\
\hline
\end{tabular}

Table 2. Condition of training dataset

In addition to this aforementioned challenge, for testing the model, the car velocity on the road is needed. It is not possible to obtain the exact velocity profile before performing the whole route. Since aggressiveness can affect the speed of the car as well, we have used the same approach to obtain the speed. The ARNN model has been trained with just slope data as input and speed as the target variable. Again one step of the speed can be used as the input for the model for the step $\mathrm{k}+1$. This training has been done for all four combinations of driving modes and road types. Figure 8 illustrates the result of this type of estimation of the speed profile for two different road types in moderate driving modes.
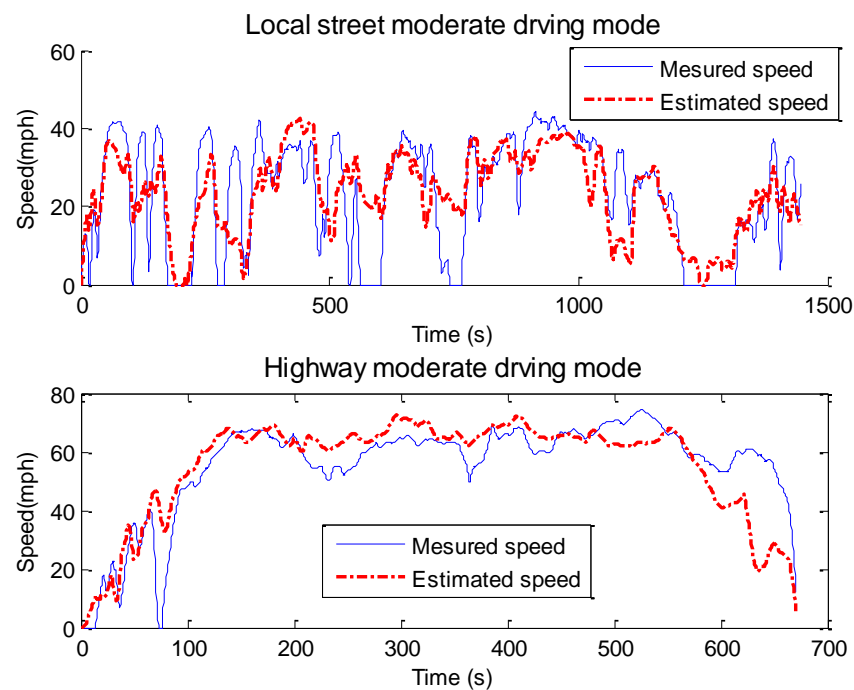

Figure 8. Estimated speed; testing for highway and local street roads

Looking at the speed predictions for the local street route shows that whenever the actual speed is zero the model cannot predict the speed accurately. The reason is that, since the model is trained based on data from another type of road, it does not have enough information from the new road except slope. Even though slope can provide some information for acceleration or deceleration of speed, it cannot give any information when the car is stopping. Some other parameters, such as the location of traffic lights and stop signs, can affect this model. But, for highway this issue does not appear and the model can provide a better estimation of the speed. The output of this model (speed) is going to be used as the input for the SoC prediction in next step.

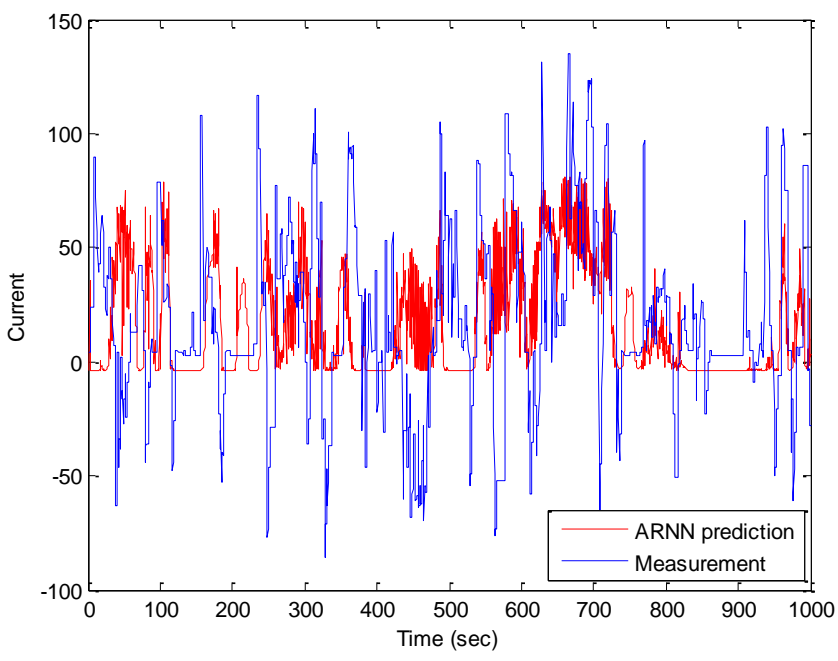

Figure 9. Comparing measured current and predictive current

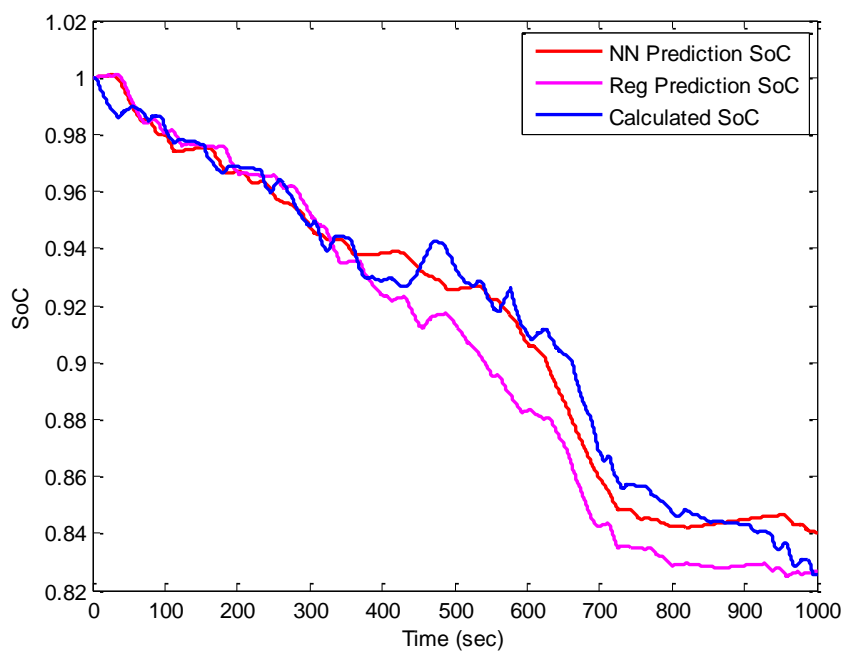

Figure 10. Comparing two methods of SoC estimation with Coloumb counting (measured)

Four different models have been trained and has been represented in Table 2. These models are the combination of each road type (local streets and highway) with one of driving modes (aggressive and moderate), so with new road data (slope of the road) the model should be able to predict the battery current profile. During testing the appropriate model will be chosen based on the types of data be it highway or local in terms of road type, and aggressive or moderate for driving behavior. Figure 9 shows the results of battery current prediction based on the second road slope and the estimated speed from the previous step with 
moderate driving mode. This estimation has been done with the ARNN model. The same approach has also been done with linear regression analysis. According to Eq. (3) the SoC can be calculated, which has been represented on Figure 10.

\section{RESULTS AND DISSCUSSION}

Figure 9 shows the behavior of the current and also shows that the prediction of the current follows the actual measurements. However, it is difficult to evaluate the accuracy of the model from the graph. Figure 10 shows that the prediction of SoC based on ARNN is accurate at certain points when compared to actual measurements, but the last value is not exactly correct. The linear regression gives a better estimation at the last point. To be able to evaluate both approaches the root mean square error has been calculated for four different testing data sets. The results have been summarized in Table 3 .

\begin{tabular}{c|c|c|c|c}
\hline & Road type & $\begin{array}{c}\text { Driving } \\
\text { mode }\end{array}$ & $\begin{array}{c}\text { RMSE } \\
\text { ARNN }\end{array}$ & $\begin{array}{c}\text { RMSE } \\
\text { Regression }\end{array}$ \\
\hline 1 & Highway 2 & Aggressive & 12.87 & 23.32 \\
\hline 2 & Highway 2 & Moderate & 10.29 & 21.21 \\
\hline 3 & Local St 2 & Aggressive & 14.32 & 25.08 \\
\hline 4 & Local St 2 & Moderate & 11.98 & 22.11 \\
\hline
\end{tabular}

Table 3. Benchmarking tests of RMSE in different road and driving condition

Comparing the results we can see that for both highway and local driving aggressive driving behavior scenarios exhibit a higher error rate in prediction. If we remember the feature extraction from driving modes, the standard deviation of the aggressive driving mode is higher which indicates that the current fluctuates much more compared to the moderate driving mode. And if the model cannot follow all of the fluctuations in current, the error increases. Another result shows that local streets have much more error in terms of prediction than Highways in the same driving modes. Based on empirical data, EVs consume a higher amount of energy in highway driving because of the higher average, though stable, speed, however, in local driving the average speed is much lower but it changes often due to traffic and stoplights, among other reasons.

Benchmarking the two algorithms (ARNN and regression) identifies accurate results from ARNN. The strength of ARNN is that they provide a dynamic modeling of the current and SoC. Figure 10 shows that SOC prediction based on ARNN follows the real values with a smaller error. However, we need to consider that this prediction has been applied on a small set of data. Larger data sets can help to enable much more accurate results.

\section{CONCLUSION}

Estimating battery state of charge is one of the most significant issues for electric vehicles. Since there are many factors that can affect battery SoC during vehicle operation, it is not easy to assess battery charge status. Discharging current rate is one of the factors that change dynamically during battery operation based on road slope, and car speed. Even if slope of the road is constant, different drivers can have disparate driving styles on the same road. In this paper a simple classification has been done on both driving modes and road types, showing how these two elements impact the current of the vehicle.

Whereas the GPS data is available in a car the GPS can show more information to the driver before in a trip. The objective is to utilize this data as the input and give the driver accurate SoC estimation, based on the different routes that the GPS provides. Two techniques have been applied in this paper. The Adaptive Recurrent Neural Network result is accurate in comparison to measured data and has a lower error rate. However, even the ARNN results still contain some error, which may be attributed to assumptions.

In fact, the method to predict the velocity profile is one of the reasons for the error. There are a variety of road condition factors that are not incorporated in this model, such as street junctions, stop signs, and traffic jams. Considering all these items in future plans can improve the speed prediction. Regardless of the velocity, this method relies on training data; the performed models are based on one road from each type. In this experiment just two roads of each type were selected. In reality there are an extremely large number of roads and road conditions. In the future it would be possible to upload data from all EVs in a cloud based system during their performance, and then apply machine learning tools easily cluster them in different regimes like different driving modes. It would then be easier to build a data driven model for prediction SoC.

\section{ACKNOWLEDGEMENT}

We would like to thank AMP Electric Vehicle Company and Mr. Don Wires for their assistance in providing infrastructure to collecting the vehicle test data.

\section{REFERENCES}

Abolhassani Monfared, N., Gharib, N., Moqtaderi, H., Hejabi, M., Amiri, M., Torabi, F., \& Mosahebi, A. (2006). Prediction of state-of-charge effects on lead-acid battery characteristics using neural network parameter modifier. Journal of Power Sources, 158(2 SPEC. ISS.), 932-935. 
Adornato, B., Patil, R., Filipi, Z., Baraket, Z., \& Gordon, T. (2009). Characterizing naturalistic driving patterns for plugin hybrid electric vehicle analysis.

Bo, C., Zhifeng, B., \& Binggang, C. (2008). State of charge estimation based on evolutionary neural network. Energy Conversion and Management, 49(10), 2788-2794.

Charkhgard, M., \& Farrokhi, M. (2010). State-of-charge estimation for lithium-ion batteries using neural networks and EKF. IEEE Transactions on Industrial Electronics, 57(12), 4178-4187.

Fodor, D., Enisz, K., Doman, R., \& Toth, P. (2011). Tire road friction coefficient estimation methods comparison based on different vehicle dynamics models.

Gonder, J., Markel, T., Thornton, M., \& Simpson, A. (2007) Using global positioning system travel data to assess real-world energy use of plug-in hybrid electric vehicles. (pp. 26-32).

He, H., Xiong, R., \& Guo, H. (2012). Online estimation of model parameters and state-of-charge of LiFePO4 batteries in electric vehicles. Applied Energy, 89(1), 413-420.

He, H., Xiong, R., Zhang, X., Sun, F., \& Fan, J. (2011). State-of-charge estimation of the lithium-ion battery using an adaptive extended Kalman filter based on an improved Thevenin model. IEEE Transactions on Vehicular Technology, 60(4), 1461-1469.

Huang, X., Tan, Y., \& He, X. (2011). An intelligent multifeature statistical approach for the discrimination of driving conditions of a hybrid electric vehicle. IEEE Transactions on Intelligent Transportation Systems, 12(2), 453-465.

Julka, N., Thirunavukkarasu, A., Lendermann, P., Gan, B. P., Schirrmann, A., Fromm, H., \& Wong, E. (2011). Making use of prognostics health management information for aerospace spare components logistics network optimisation. Computers in Industry, 62(6), 613-622.

Khaled, M., Harambat, F., Yammine, A., \& Peerhossaini, H. (2010). Aerodynamic forces on a simplified car body - Towards innovative designs for car drag reduction.

Lee, T. K., Baraket, Z., Gordon, T., \& Filipi, Z. (2011). Characterizing One-day Missions of PHEVs Based on Representative Synthetic Driving Cycles. SAE International Journal of Engines, 4(1), 1088-1101.

Liaw, B. Y., \& Dubarry, M. (2007). From driving cycle analysis to understanding battery performance in real-life electric hybrid vehicle operation. Journal of Power Sources, 174(1), 76-88.

MacLean, H. L., \& Lave, L. B. (2003). Life Cycle Assessment of Automobile/Fuel Options. Environmental Science and Technology, 37(23), 5445-5452.
Marina de Queiroz Tavares, J. G., Flavio Perucchi, Franz Baumgartner, Maria Youssefzadeh. (2010). Understanding future customer needs by monitoring EV-drivers' behavior. Paper presented at the Hybrid and Fuel Cell Electric Vehicle Symposium \& Exhibition, Shenzhen, China.

Meissner, E., \& Richter, G. (2003). Battery Monitoring and Electrical Energy Management precondition for future vehicle electric power systems. Journal of Power Sources, 116(1-2), 79-98.

Montazeri-Gh, M., Fotouhi, A., \& Naderpour, A. (2011). Driving patterns clustering based on driving feature analysis. Proceedings of the Institution of Mechanical Engineers, Part C: Journal of Mechanical Engineering Science, 225(6), 13011317.

Piller, S., Perrin, M., \& Jossen, A. (2001). Methods for state-of-charge determination and their applications. Journal of Power Sources, 96(1), 113-120.

Rodrigues, S., Munichandraiah, N., \& Shukla, A. K. (2000). Review of state-of-charge indication of batteries by means of a.c. impedance measurements. Journal of Power Sources, 87(1), 12-20.

Shafiei, A., \& Williamson, S. S. (2010). Plug-in hybrid electric vehicle charging: Current issues and future challenges.

Shukla, A. K., Aricò, A. S., \& Antonucci, V. (2001). An appraisal of electric automobile power sources. Renewable and Sustainable Energy Reviews, 5(2), 137-155.

Sioshansi, R., \& Denholm, P. (2009). Emissions impacts and benefits of plug-in hybrid electric vehicles and vehicle-to-grid services. Environmental Science and Technology, 43(4), 1199-1204.

Ulrich, L. (2012). State of charge. IEEE Spectrum, 49(1), 56-59.

Wang, W. Q., Golnaraghi, M. F., \& Ismail, F. (2004). Prognosis of machine health condition using neurofuzzy systems. Mechanical Systems and Signal Processing, 18(4), 813-831.

$\mathrm{Xu}$, L., Wang, J., \& Chen, Q. (2012). Kalman filtering state of charge estimation for battery management system based on a stochastic fuzzy neural network battery model. Energy Conversion and Management, 53(1), 33-39.

Zhang, J., \& Lee, J. (2011). A review on prognostics and health monitoring of Li-ion battery. Journal of Power Sources, 196(15), 6007-6014.

Zhu, C. B., Coleman, M., \& Hurley, W. G. (2004). State of charge determination in a lead-acid battery: Combined EMF estimation and Ah-balance approach. 